\title{
Prevention of neurological complications using a neural monitoring system with a finger electrode in the extreme lateral interbody fusion approach
}

\author{
Wataru Narita, MD, ${ }^{1,2}$ Ryota Takatori, MD, PhD, ${ }^{1}$ Yuji Arai, MD, PhD, ${ }^{1}$ Masateru Nagae, MD, PhD, ${ }^{1}$ \\ Hitoshi Tonomura, MD, PhD, ${ }^{1}$ Tatsuro Hayashida, MD, PhD, ${ }^{2}$ Taku Ogura, MD, PhD, ${ }^{2}$ \\ Hiroyoshi Fujiwara, MD, PhD, ${ }^{1}$ and Toshikazu Kubo, MD, PhD' \\ 'Department of Orthopaedics, Graduate School of Medical Science, Kyoto Prefectural University of Medicine; and 'Spine \\ Surgery and Related Research Center, Nantan General Hospital, Nantan City, Kyoto, Japan
}

\begin{abstract}
OBJECTIVE Extreme lateral interbody fusion (XLIF) is a minimally disruptive surgical procedure that uses a lateral approach. There is, however, concern about the development of neurological complications when this approach is used, particularly at the L4-5 level. The authors performed a prospective study of the effects of a new neural monitoring system using a finger electrode to prevent neurological complications in patients treated with XLIF and compared the results to results obtained in historical controls.
\end{abstract}

METHODS The study group comprised 36 patients (12 male and 24 female) who underwent XLIF for lumbar spine degenerative spondylolisthesis or lumbar spine degenerative scoliosis at $L 4-5$ or a lower level. Using preoperative axial MR images obtained at the mid-height of the disc at the treated level, we calculated the psoas position value (PP\%) by dividing the distance from the posterior border of the vertebral disc to the posterior border of the psoas major muscle by the anteroposterior diameter of the vertebral disc. During the operation, the psoas major muscle was dissected using an index finger fitted with a finger electrode, and threshold values of the dilator were recorded before and after dissection. Eighteen cases in which patients had undergone the same procedure for the same indications but without use of the finger electrode served as historical controls. Baseline clinical and demographic characteristics, PP values, clinical results, and neurological complications were compared between the 2 groups.

RESULTS The mean PP\% values in the control and finger electrode groups were $17.5 \%$ and $20.1 \%$, respectively (no significant difference). However, 6 patients in the finger electrode group had a rising psoas sign with PP\% values of $50 \%$ or higher. The mean threshold value before dissection in the finger electrode group was $13.1 \pm 5.9 \mathrm{~mA}$, and this was significantly increased to $19.0 \pm 1.5 \mathrm{~mA}$ after dissection $(p<0.001)$. A strong negative correlation was found between $\mathrm{PP} \%$ and threshold values before dissection, but there was no correlation with threshold values after dissection. The thresholds after dissection improved to $11 \mathrm{~mA}$ or higher in all patients. There were no serious neurological complications in any patient, but there was a significantly lower incidence of transient neurological symptoms in the finger electrode group ( 7 [38\%] of 18 cases vs 5 [14\%] of 36 cases, $p=0.047$ ).

CONCLUSIONS The new neural monitoring system using a finger electrode may be useful to prevent XLIF-induced neurological complications.

http://thejns.org/doi/abs/10.3171/2016.1.SPINE151069

KEY WORDS neural monitoring; extreme lateral interbody fusion; XLIF; complications; finger electrode; minimally invasive spine stabilization; lumbar

A NTERIOR lumbar interbody fusion (ALIF) is the common anterior approach for lumbar fixation, and posterior lumbar interbody fusion (PLIF) and transforaminal lumbar interbody fusion (TLIF) are com- mon posterior approaches. Difficulty with exposure is a problem with the anterior approach, whereas invasion of the paraspinal muscles and the insertable cage size are problematic in the posterior approach. In contrast, the

ABBREVIATIONS ALIF = anterior lumbar interbody fusion; BLN-VAS = VAS for gluteal and lower limb numbness; BLP-VAS= VAS for gluteal and lower-limb pain; BMI = body mass index; JOA = Japanese Orthopaedic Association; LBP-VAS = VAS for low-back pain; PP\% = psoas position (expressed as a percentage); VAS = visual analog scale; XLIF = extreme lateral interbody fusion.

SUBMITTED September 9, 2015. ACCEPTED January 26, 2016.

INCLUDE WHEN CITING Published online May 20, 2016; DOI: 10.3171/2016.1.SPINE151069. 
lateral approach via a retroperitoneal pathway allows the vertebral body and intervertebral disc to be reached directly, and extreme lateral interbody fusion (XLIF, NuVasive, Inc.) was reported as an effective and minimally disruptive surgical procedure for anterior lumbar fusion in $2006 .{ }^{15}$ In XLIF, the psoas major muscle is split while the patient is in the lateral position, and a large cage is fixed between the vertebrae. This procedure is useful for obtaining effective reduction and favorable alignment using a large cage through a minimally disruptive approach and indirect decompression of the spinal canal and intervertebral foramen., ${ }^{4,14}$

There are concerns, however, about the high rate of neurological complications associated with the lateral approach, particularly for surgery at the L4-5 level or lower, due to the anatomical structure of the lumbar plexus (the psoas major muscle containing the nerve plexus being present in the anterior region). ${ }^{6,12,18,20,23,25}$ Therefore, neural monitoring is required for XLIF. ${ }^{15,19,23}$ A dilator equipped with an electrode is inserted toward the vertebral region through the psoas major muscle while the threshold displayed on the monitor is observed. ${ }^{19}$ Such surgery should be suspended when the insertion route is close to the lumbar plexus because of the invasive risk to the lumbar plexus. ${ }^{24}$ This concern may lead to XLIF being excluded in preoperative planning.

To overcome these problems, we have developed an electrode that can be connected to a conventional neural monitoring system and also can be attached to the surgeon's index finger for atraumatic dissection of the perineural tissue through the XLIF approach while monitoring the nerve. During neural monitoring this finger electrode may provide greater protection from nerve damage than the dilator electrode alone. This may allow XLIF to be performed safely, and the procedure may become applicable in patients for whom it would not otherwise be an option. The objectives of this study were to describe the procedure for neural monitoring using a finger electrode in the XLIF approach and to investigate its effect on prevention of neurological complications.

\section{Methods \\ Patients}

The study group included 36 patients (12 male, 24 female) who were treated with XLIF for lumbar disease at L4-5 or a lower level between April and October 2014. The patients' mean age was 71.5 years (range 54-80 years), and their mean body mass index (BMI) was 23.1 $\mathrm{kg} / \mathrm{m}^{2}$. The affected vertebral level was L4-5 in 34 cases and L5-6 in 2. Twenty-one of the patients had degenerative lumbar spondylolisthesis, and 15 had degenerative lumbar scoliosis. Surgery was indicated for degenerative spondylolisthesis with a sagittal translation $\geq 3 \mathrm{~mm}$ on ante- and retroflexion in lateral radiographs obtained in a standing position or $\mathrm{a} \geq 5^{\circ}$ intervertebral wedge shape on anteflexion and for degenerative scoliosis with a Cobb angle $\geq 10^{\circ}$ in the anteroposterior radiographs obtained in a standing position in combination (for both conditions) with intolerable lower limb neurological manifestations resistant to conservative treatment for several months. Pa- tients with a history of lumbar surgery were included in the study, but those who had motor paralysis of the lower extremities before surgery were excluded. All patients who met the inclusion criteria and were treated with XLIF using the finger electrode during the study period were included. Consent for use of the finger electrode and study participation was obtained from all participants. The finger electrode was used for almost all cases during the study period. However, in some cases, the device was not ready for use and the patients involved in those cases were not included in the study.

Eighteen consecutive patients ( 7 male, 11 female) who underwent XLIF from November 2013 to April 2014 (without use of the finger electrode) were included as historical controls. These patients were chosen because they were the last 18 patients treated with XLIF before we began to use the finger electrode. Their mean age was 71.5 years (range 54-83 years), and their mean BMI was 23.9 $\mathrm{kg} / \mathrm{m}^{2}$. The treated level was L4-5 in all 18 cases. Twelve of the patients had degenerative lumbar spondylolisthesis and 6 had degenerative lumbar scoliosis.

\section{Finger Electrode}

The finger electrode was designed to allow connection to a neural monitoring system (NVM5, NuVasive, Inc. $)^{19}$ that is used during spinal surgery, and it measures the current threshold in the same way as the dilator electrode that is usually used with the NVM5. The mean resistance between the finger electrode and the monitor connection of NVM5 is $\leq 0.1 \Omega$, and the conductivity is equivalent to that of the dilator electrode. The system comprises a silicone rubber part attached to the fingertip (outer diameter 18 $\mathrm{mm}$, inner diameter $17 \mathrm{~mm}$, thickness $1 \mathrm{~mm}$ ), a siliconecovered tin-plated copper cable, and an aluminum section connecting the cable to the NVM5 (diameter $6 \mathrm{~mm}$ ). The finger pulp surface of the fingertip part is equipped with a silver electrode $(\varphi 6 \times 4 \mathrm{~mm})$ (Fig. 1). All components are made of sterilizable materials and are compatible with autoclaves, plasma sterilization, and ethylene oxide sterilization. The finger electrode was designed in our laboratory and is manufactured by Umihira Co., Ltd. It is commercially available in Japan (Product No. UM-232-001).

\section{Surgical Procedure}

Surgery is performed with the patient in a lateral position. The operating table is slightly bent at the iliac region. ${ }^{15}$ Marking is applied under fluoroscopy, and an oblique incision of about $4 \mathrm{~cm}$ is made in the lateral abdomen. The external and internal oblique abdominal muscles and transverse abdominal muscle are bluntly exposed using the finger, and identification of the psoas major muscle is confirmed. Under fluoroscopy, the dilator connected to the NVM5 neural monitoring system is inserted into the central region of the intervertebral disc through the psoas major muscle, and the threshold of the region anterior to the dilator is recorded (pre-dissection threshold). The dilator is removed, the index finger with the electrode attached toward the finger pad is inserted through the incision, and fluoroscopy is used to confirm the location of the electrode. The psoas major muscle is then dissected un- 

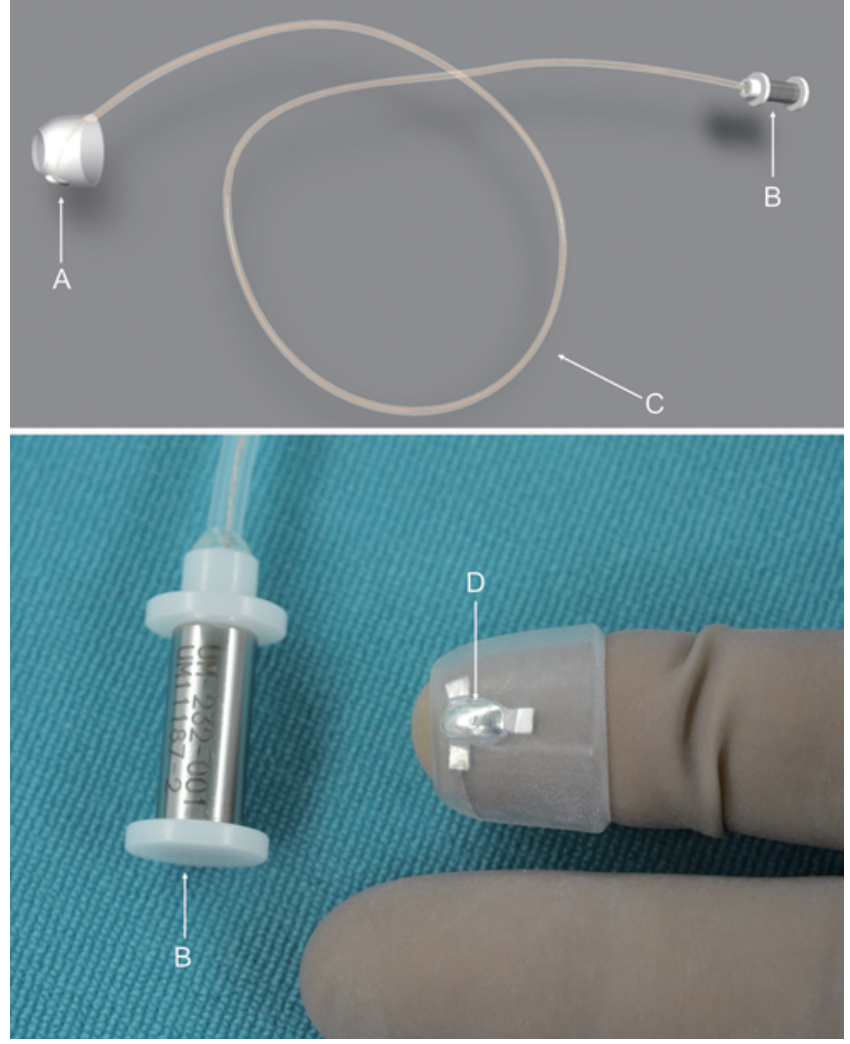

FIG. 1. Photographs of components of the finger electrode system used in this study, including the finger attachment (outer diameter $18 \mathrm{~mm}$, inner diameter $17 \mathrm{~mm}$, thickness $1 \mathrm{~mm}$, made of silicone rubber), indicated by $A$; the connection to the neural monitoring system (diameter 6 $\mathrm{mm}$, made of aluminum), indicated by $B$; the cable connecting the finger attachment to the connector (silicone-covered, tin-plated copper wire), indicated by $\mathrm{C}$; and the electrode $(6 \times 4-\mathrm{mm}$ oval, silver $)$ at the pulp surface of the tip of the finger attachment, indicated by $D$. Figure is available in color online only.

der neural monitoring performed by rotating and moving the index finger craniocaudally and anteroposteriorly. The psoas major muscle is then bluntly dissected from the intervertebral disc backward. After dissection, the dilator is inserted along the dorsal side of the index finger (Video 1).

VIDEO 1. Animated illustration showing the use of the finger electrode used in this study. Dissection can be performed with a dilator, but we believe that such dissection can be performed more safely with the use of a finger. An additional benefit over the use of a dilatator is that use of a finger provides the surgeon with a direct feel of the tissue. Copyright Wataru Narita. Published with permission.

Click here to view.

The index finger is pulled out while the dilator is left in place, and the threshold of the region anterior to the dilator is recorded again (post-dissection threshold) (Fig. 2).

Patients in the control group underwent XLIF without use of the finger electrode. The procedure was otherwise identical to that described above.

\section{Investigation Items}

The operative time required for the anterior procedure, intraoperative blood loss, and clinical outcome were investigated. Clinical outcome was evaluated using the

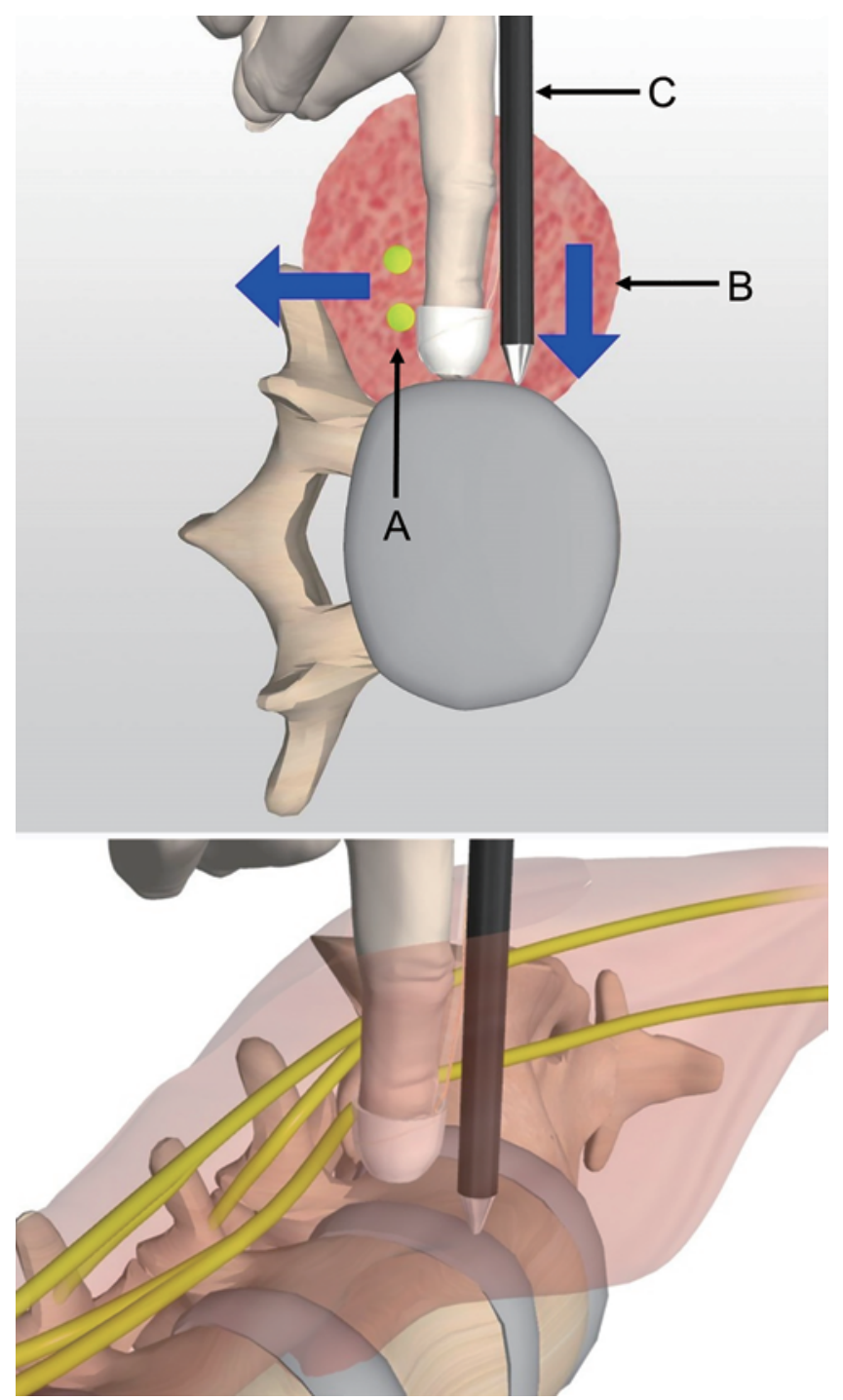

FIG. 2. Schematic diagram of the intraoperative procedure. While adjusting the direction and force of the fingertip, the psoas major muscle $(B)$ is bluntly dissected from the intervertebral disc backward. After dissection, the dilator $(\mathrm{C})$ is inserted along the dorsal side of the index finger. A indicates the lumbar plexus.

Japanese Orthopaedic Association (JOA) score, and visual analog scales ${ }^{5,8,10}$ for low-back pain, gluteal and lower limb pain, and gluteal and lower limb numbness.

Lumbar MRI (Philips Gyroscan NT Intera 1.5 T) was performed before surgery. Using axial T2-weighted MR images obtained at the central level of the intervertebral disc, the distance $(\mathrm{P})$ between the posterior margin of the intervertebral disc and posterior border of the psoas major muscle was divided by the anteroposterior diameter (D) of the intervertebral disc to calculate the psoas position (PP\%): $\mathrm{PP} \%=\mathrm{P} / \mathrm{D} \times 100$ (Fig. 3). Cases in which the posterior border of the psoas major muscle went over the center of the intervertebral disc $(\mathrm{PP} \% \geq 50 \%)$ were defined as rising psoas sign-positive. Patients were divided into those with pre-dissection thresholds measured using the dilator electrode of $\leq 10 \mathrm{~mA}$ and $>10 \mathrm{~mA} \cdot{ }^{19}$ Femoral pain/ numbness and muscle weakness on the approach side that 


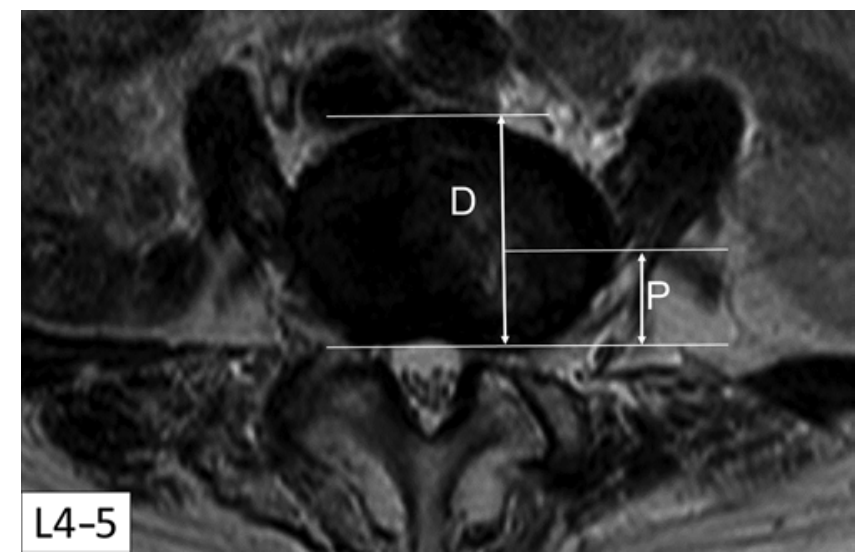

FIG. 3. Measurement of psoas position. Using data from axial T2weighted MRI images of the center of the intervertebral disc, the distance $(P)$ between the posterior margin of the intervertebral disc and the posterior border of the psoas major muscle was divided by the anteroposterior diameter (D) of the intervertebral disc. This value was defined as PP\% (PP\% = P/D × 100).

continued for 1 week or longer after surgery were regarded as neurological complications induced by the approach. The correlation between the duration of these neurological symptoms and $\mathrm{PP} \%$ was investigated.

\section{Statistical Analysis}

Comparisons between the finger electrode and control groups were performed by means of a Mann-Whitney Utest for age, $\mathrm{BMI}, \mathrm{PP} \%$, anterior surgical time, and intraoperative blood loss, and by Fisher exact test for sex, target intervertebral region, and neurological complications. The threshold values before and after dissection in the finger electrode group were examined by means of the Wilcoxon signed-rank test. Spearman rank correlation analysis was used to compare threshold values, $\mathrm{PP} \%$, the duration of neurological symptoms, and correlation with PP values in the finger electrode group before and after dissection. Power analysis was used to evaluate the required sample size. All analyses were performed using SPSS version 23.0 (IBM).

\section{Institutional Ethics Committee Approval}

Approval was obtained from the ethics committee of Nantan General Hospital for the prospective study of the use of the finger electrode.

\section{Results}

\section{Baseline Characteristics of the Patient Groups}

Age, sex, BMI, target intervertebral region, intraoperative blood loss in anterior procedures, and surgical time are shown in Table 1. None of these items differed significantly between the controls and the finger electrode group.

\section{Position of the Psoas Major Muscle Relative to the Intervertebral Disc}

The mean PP\% was $20 \% \pm 18.8 \%(0 \%-60 \%)$ in the finger electrode group and $17.5 \% \pm 14.2 \%(0 \%-41 \%)$ in the
TABLE 1. Characteristics of patients who underwent XLIF with use of the finger electrode and historical controls*

\begin{tabular}{lccc}
\hline \multicolumn{1}{c}{ Characteristic } & $\begin{array}{c}\text { Finger Electrode } \\
\text { Group }(\mathrm{n}=36)\end{array}$ & $\begin{array}{c}\text { Controls } \\
(\mathrm{n}=18)\end{array}$ & $\mathrm{p}$ Value \\
\hline Age in yrs & & & \\
\hline Mean & 71.5 & 71.5 & 0.721 \\
\hline Range & $54-80$ & $54-83$ & \\
\hline Sex: M/F & $12: 24$ & $7: 11$ & 0.573 \\
\hline BMI (kg/m $)$, mean & 23.1 & 23.9 & 0.687 \\
\hline Level treated & & & \\
\hline L1-2 & 2 & & \\
\hline L2-3 & 5 & 7 & \\
\hline L3-4 & 13 & 8 & \\
\hline L4-5 & 34 & 18 & \\
\hline $\begin{array}{l}\text { L5-6 } \\
\text { PP\%, mean }\end{array}$ & 2 & 17.5 & 0.244 \\
\hline $\begin{array}{l}\text { Intraop blood loss } \\
\text { (mL), mean }\end{array}$ & 20.1 & 18.0 & 0.328 \\
\hline $\begin{array}{l}\text { Op time (mins), mean } \\
\text { per level }\end{array}$ & 13.1 & 72.7 & 0.385 \\
\hline
\end{tabular}

* Values are numbers of patients where not otherwise indicated.

control group. The PP\% values did not differ significantly between the 2 groups. However, no rising psoas sign (50\% or higher) was seen in the control group, whereas a rising psoas sign occurred in 6 of the 36 patients in the finger electrode group-at L4-5 in 5 of 34 patients and at L5-6 in 1 of 2 patients.

\section{Correlation of PP\% With Thresholds Before and After Dissection in Finger Electrode Group}

The mean pre-dissection threshold was $13.1 \pm 5.9 \mathrm{~mA}$ (1-20 mA), and the mean threshold measured after dissection with the finger electrode was $19.0 \pm 1.5 \mathrm{~mA}(14-20$ $\mathrm{mA})$, showing a significant increase $(\mathrm{p}<0.001) . \mathrm{PP} \%$ and the pre-dissection threshold were inversely correlated (rho $=-0.60, p<0.001)$, but there was no correlation between $\mathrm{PP} \%$ and the post-dissection threshold (rho $=-0.159, \mathrm{p}$ $=0.04$ ). The pre-dissection threshold was $\leq 10 \mathrm{~mA}$ in 7 patients, and PP\% was relatively high (45\%) in these patients, since the psoas major muscle was present in the anterior region to a greater extent than that in patients with a pre-dissection threshold of $\geq 11 \mathrm{~mA}$. The post-dissection threshold was $\geq 11 \mathrm{~mA}$ in all patients (Fig. 4).

\section{Clinical Outcome}

Substantial improvement was evident in the mean scores for all outcome measures in both groups of patients (Table 2). The rate of postoperative neurological complications was significantly higher in the control group than in the finger electrode group, with complications occurring in 7 (38\%) of 18 patients in the control group and $5(14 \%)$ of 36 patients in the finger electrode group $(p<0.05)$. In all cases, however, the symptoms resolved within 3 weeks, and no serious neurological complication developed (Fig. 5). There was no significant correlation between PP\% and the duration of neurological manifestations. 

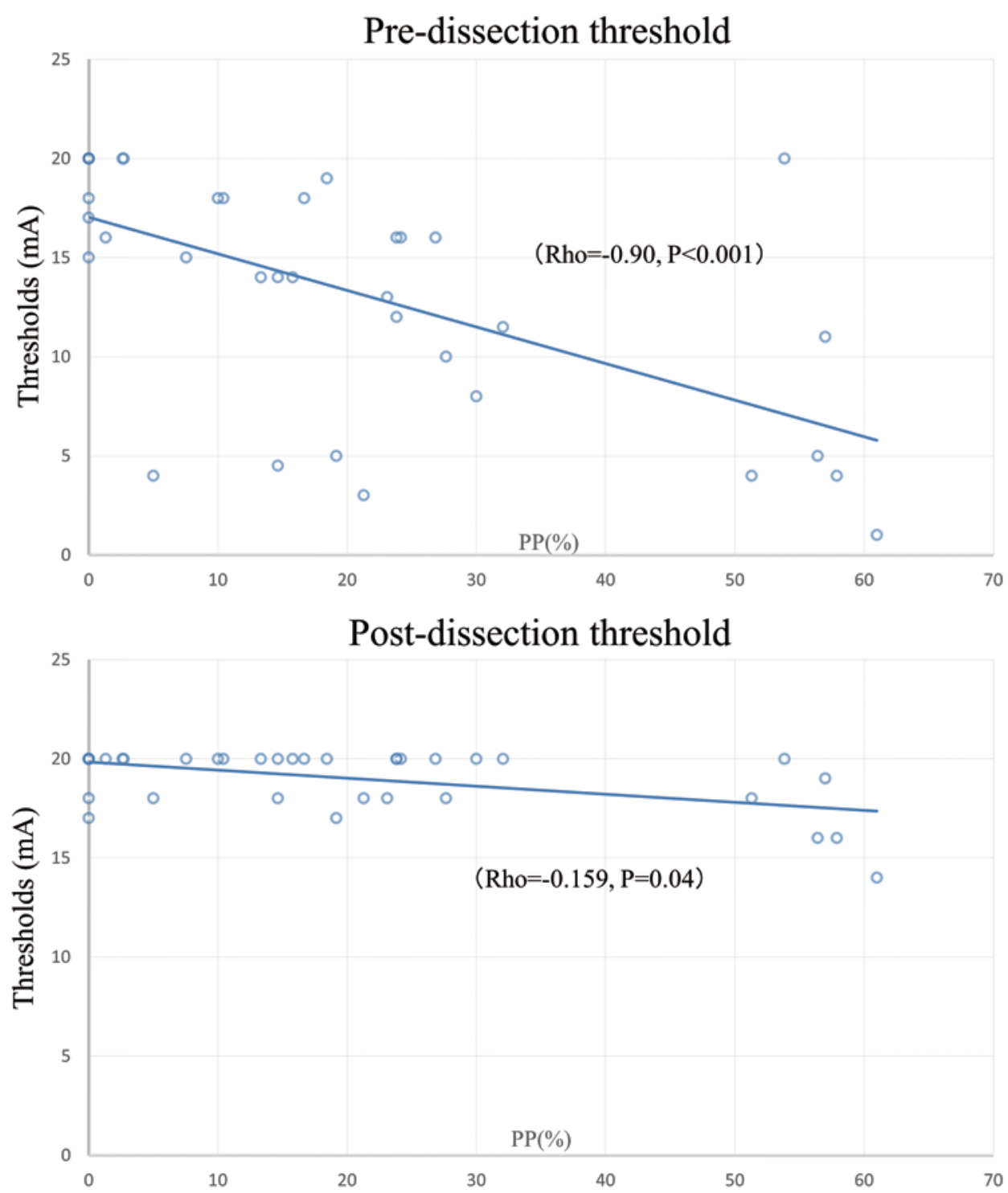

FIG. 4. Plots of $\mathrm{PP} \%$ against thresholds before and after dissection. PP\% and the pre-dissection threshold were inversely correlated (rho $=-0.60, p<0.001$ ). There was no correlation between PP\% and the post-dissection threshold (rho $=-0.159, p=0.04$ ). Figure is available in color online only.

\section{Discussion}

XLIF is a minimally disruptive surgical procedure that is effective for anterior fusion but has the risk of neurological complications due to use of a lateral approach. ${ }^{-3,9,12,16-18,21,24}$ Reduction of neurological complications is likely to improve the surgical outcome of XLIF and expand the indication. For this reason, we developed a new neural monitoring system using a finger electrode to reach the intervertebral region in patients undergoing XLIF. The results of the current study show that this method is effective for preventing neurological complications.

In XLIF, the psoas major muscle containing the lumbar plexus is exposed to reach the intervertebral region, and the risk of neurological complications is high when this muscle is present in an anterior position. . $^{12,13,18,22,23,25}$ The position of the psoas major muscle relative to the interver- tebral disc is a useful index of the positional relationship between the nerve and the muscle. ${ }^{25} \mathrm{~A}$ case in which the posterior border of the psoas major muscle is anterior to the center of the anteroposterior diameter of the intervertebral disc is defined as having a rising psoas sign, and the lateral approach is difficult in such cases. ${ }^{24}$ The rising psoas sign is common at L4-5 and L5-6, and in many of these cases the patients cannot be treated with a conventional XLIF procedure. ${ }^{18,24,25}$

In this study, the positional relationship between the psoas major muscle and nerve was quantified using the psoas position ( $\mathrm{PP} \%$ ) calculated by dividing the distance (P) between the posterior margin of the intervertebral disc and posterior border of the psoas major muscle by the anteroposterior diameter (D) of the intervertebral disc, based on data from preoperative axial MR images of the inter- 
vertebral disc. The PP $\%$ was $\geq 50 \%$ (a rising psoas sign) in 6 of the 36 patients in whom surgery was performed using the finger electrode, indicating a significant correlation between PP\% and the pre-dissection threshold of the initially inserted dilator electrode. As previously found, the distance between the psoas major muscle and nerve is short at L4-5 and L5-6, and the nerve becomes closer to the muscle as the psoas major muscle position becomes anterior in the cross-sectional view of the intervertebral region on preoperative MRI. The nerve should be sufficiently retracted at these levels to reduce the risk of neurological complications. . $^{18,20,23,25}$

Sufficient dissection of the nerve using the XLIF approach with a dilator alone is difficult, even with neural monitoring. In contrast, the procedure using the finger electrode system is atraumatic because the operator's fin- ger, attached to the electrode, reaches the intervertebral region while monitoring the nerve. The L4-5 and L5-6 intervertebral regions were approached using the finger electrode system in patients with degenerative lumbar spondylolisthesis and degenerative lumbar scoliosis, and the threshold significantly rose after dissection with the finger electrode. In addition, the post-dissection threshold was not correlated with the position of the psoas major muscle relative to the intervertebral disc. The threshold improved to $\geq 11 \mathrm{~mA}$ in all patients, including the 6 cases in which the rising psoas sign was present, with $\mathrm{PP} \% \geq$ $50 \%$, reducing the risk of nerve damage. Based on these findings, the nerve can be atraumatically dissected using the finger electrode system, even if the psoas major muscle and nerve are close to each other, and the intervertebral region can be safely reached.
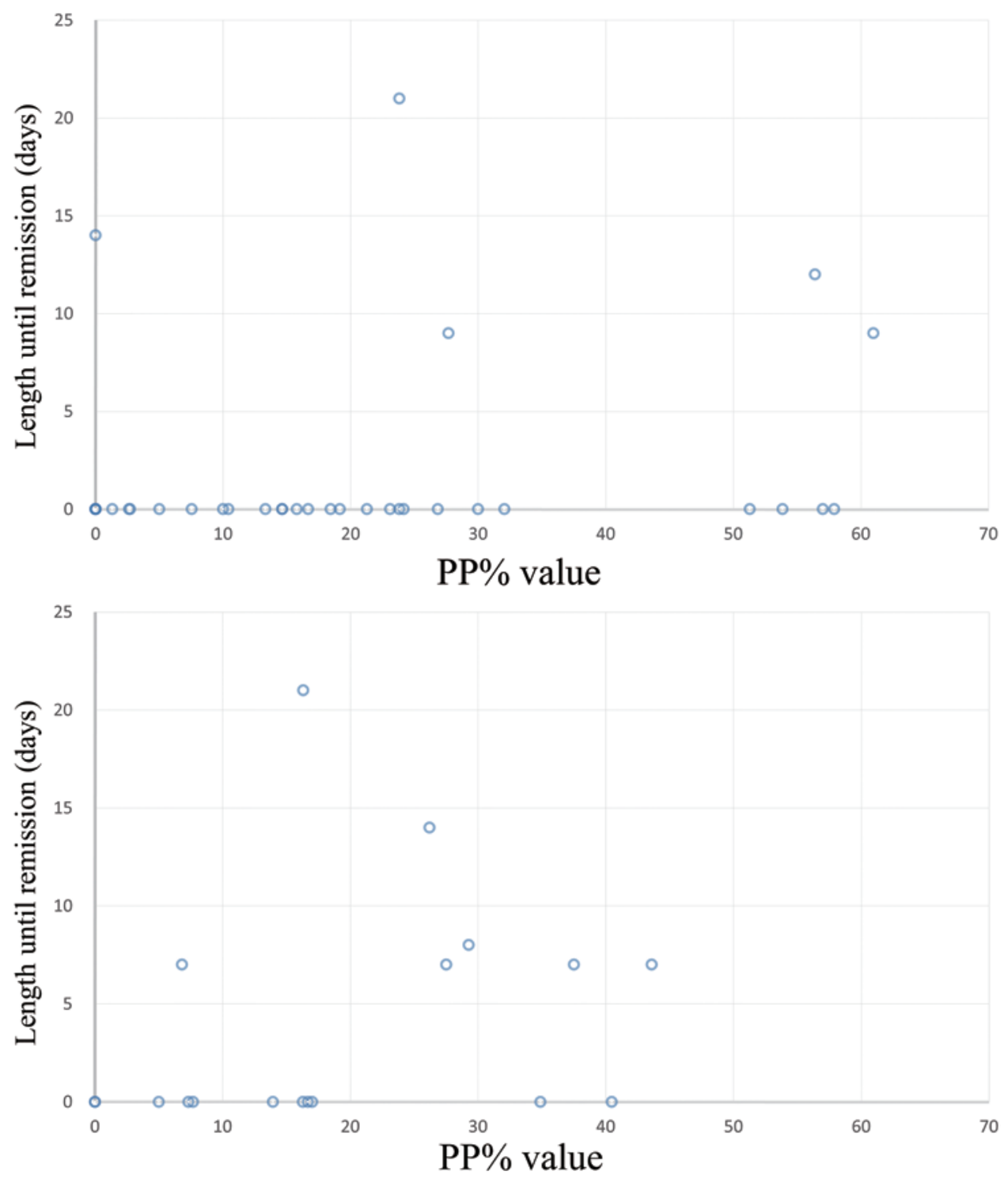

FIG. 5. Plot of PP\% against the duration of induced neurological manifestations. There was no significant correlation between the iliopsoas muscle position and duration. Symptoms remitted within 3 weeks and no serious neurological complication developed in any patients. Upper: Finger electrode group. Lower: Control group. Figure is available in color online only. 
TABLE 2. Clinical outcome*

\begin{tabular}{cccc}
\hline Variable & $\begin{array}{c}\text { Finger Electrode } \\
\text { Group }(n=36)\end{array}$ & Controls $(n=18)$ & $p$ Value \\
\hline JOA score & & & \\
\hline Preop & $13.9 \pm 7.5$ & $14.6 \pm 7.2$ & 0.411 \\
\hline Postop & $23.1 \pm 6.2$ & $22.4 \pm 5.9$ & 0.375 \\
\hline LBP-VAS (mm) & & & \\
\hline Preop & $54.8 \pm 24.1$ & $59.2 \pm 27.2$ & 0.545 \\
\hline Postop & $15.4 \pm 11.5$ & $12.9 \pm 10.5$ & 0.492 \\
\hline BLP-VAS (mm) & & & \\
\hline Preop & $46.4 \pm 20.1$ & $43.2 \pm 17.2$ & 0.725 \\
\hline Postop & $11.2 \pm 9.9$ & $13.4 \pm 10.1$ & 0.333 \\
\hline BLN-VAS (mm) & & & \\
\hline Preop & $44.1 \pm 18.5$ & $49.6 \pm 17.2$ & 0.466 \\
\hline Postop & $19.2 \pm 9.5$ & $20.4 \pm 10.9$ & 0.61 \\
\hline No. of pts w/ neural & $5(14 \%)$ & $7(38 \%)$ & $0.047 \dagger$ \\
complications & & & \\
\hline
\end{tabular}

JOA = Japanese Orthopaedic Association; pts = patients.

* Values are means and standard deviations unless otherwise indicated.

† Significant difference in a Fisher exact test.

The limitations of this study include the possibility that the finger electrode cannot be used because the surgeon's finger might not reach the vertebral disc in a patient with a large build. In addition, $\mathrm{PP} \%$ values obtained in the supine position in MRI before surgery were used to show the location of the psoas major muscle, but the perioperative location and tone of this muscle might differ from those in MRI because the hip is flexed in the lateral position during XLIF.

The incidence of XLIF-induced postoperative neurological manifestations is $1 \%-60 \%,{ }^{24}$ with causes including direct subcutaneous and between-muscle procedures, compression by retractors, and postoperative hematoma. ${ }^{2,3,7,9,11,12,17,21,24}$ No serious neurological complications occurred in any patients in the current study, but the incidence of neurological complications of $38.9 \%$ in the conventional group was comparatively high, as also seen in past reports. In contrast, the rate of neurological complications of 13\% (5 in 36 patients) in the finger electrode group was significantly lower than that in the control group. These results indicate that neural monitoring using a finger electrode is effective for prevention of postoperative neurological manifestations induced by XLIF. The finger electrode may also be useful in other procedures in which neurological complications may occur.

\section{Acknowledgments}

We are grateful to Umihira Co., Ltd., for assistance with development of the finger electrode and to members of the Society for Minimally Invasive Spine Stabilization (MISt) for their advice.

\section{References}

1. Ahmadian A, Abel N, Uribe JS: Functional recovery of severe obturator and femoral nerve injuries after lateral retroperitoneal transpsoas surgery. J Neurosurg Spine 18:409414,2013
2. Ahmadian A, Deukmedjian AR, Abel N, Dakwar E, Uribe JS: Analysis of lumbar plexopathies and nerve injury after lateral retroperitoneal transpsoas approach: diagnostic standardization. J Neurosurg Spine 18:289-297, 2013

3. Ahmadian A, Verma S, Mundis GM Jr, Oskouian RJ Jr, Smith DA, Uribe JS: Minimally invasive lateral retroperitoneal transpsoas interbody fusion for L4-5 spondylolisthesis: clinical outcomes. J Neurosurg Spine 19:314-320, 2013

4. Alimi M, Hofstetter CP, Cong GT, Tsiouris AJ, James AR, Paulo D, et al: Radiological and clinical outcomes following extreme lateral interbody fusion. J Neurosurg Spine 20:623-635, 2014

5. Azimi P, Shahzadi S, Montazeri A: The Japanese Orthopedic Association Back Pain Evaluation Questionnaire (JOABPEQ) for low back disorders: a validation study from Iran. J Orthop Sci 17:521-525, 2012

6. Benglis DM, Vanni S, Levi AD: An anatomical study of the lumbosacral plexus as related to the minimally invasive transpsoas approach to the lumbar spine. J Neurosurg Spine 10:139-144, 2009

7. Dakwar E, Cardona RF, Smith DA, Uribe JS: Early outcomes and safety of the minimally invasive, lateral retroperitoneal transpsoas approach for adult degenerative scoliosis. Neurosurg Focus 28(3):E8, 2010

8. Fukui M, Chiba K, Kawakami M, Kikuchi S, Konno S, Miyamoto M, et al: JOA Back Pain Evaluation Questionnaire (JOABPEQ)/JOA Cervical Myelopathy Evaluation Questionnaire (JOACMEQ). The report on the development of revised versions. April 16, 2007. J Orthop Sci 14:348-365, 2009

9. Grimm BD, Leas DP, Poletti SC, Johnson DR II: Postoperative complications within the first year after extreme lateral interbody fusion: experience of the first 108 patients. J Spinal Disord Tech [epub ahead of print], 2014

10. Hashizume H, Konno S, Takeshita K, Fukui M, Takahashi K, Chiba K, et al: Japanese orthopaedic association back pain evaluation questionnaire (JOABPEQ) as an outcome measure for patients with low back pain: reference values in healthy volunteers. J Orthop Sci 20:264-280, 2015

11. Isaacs RE, Hyde J, Goodrich JA, Rodgers WB, Phillips FM: A prospective, nonrandomized, multicenter evaluation of extreme lateral interbody fusion for the treatment of adult degenerative scoliosis: perioperative outcomes and complications. Spine (Phila Pa 1976) 35 (26 Suppl):S322-S330, 2010

12. Le TV, Burkett CJ, Deukmedjian AR, Uribe JS: Postoperative lumbar plexus injury after lumbar retroperitoneal transpsoas minimally invasive lateral interbody fusion. Spine (Phila Pa 1976) 38:E13-E20, 2013

13. Menezes CM, de Andrade LM, Herrero CF, Defino HL, Ferreira Júnior MA, Rodgers WB, et al: Diffusion-weighted magnetic resonance (DW-MR) neurography of the lumbar plexus in the preoperative planning of lateral access lumbar surgery. Eur Spine J 24:817-826, 2015

14. Oliveira L, Marchi L, Coutinho E, Pimenta L: A radiographic assessment of the ability of the extreme lateral interbody fusion procedure to indirectly decompress the neural elements. Spine (Phila Pa 1976) 35 (26 Suppl):S331-S337, 2010

15. Ozgur BM, Aryan HE, Pimenta L, Taylor WR: Extreme lateral interbody fusion (XLIF): a novel surgical technique for anterior lumbar interbody fusion. Spine J 6:435-443, 2006

16. Rodgers WB, Cox CS, Gerber EJ: Early complications of extreme lateral interbody fusion in the obese. J Spinal Disord Tech 23:393-397, 2010

17. Rodgers WB, Gerber EJ, Patterson J: Intraoperative and early postoperative complications in extreme lateral interbody fusion: an analysis of 600 cases. Spine (Phila Pa 1976) 36:2632, 2011

18. Smith WD, Youssef JA, Christian G, Serrano S, Hyde JA: Lumbarized sacrum as a relative contraindication for lateral 
transpsoas interbody fusion at L5-6. J Spinal Disord Tech 25:285-291, 2012

19. Tohmeh AG, Rodgers WB, Peterson MD: Dynamically evoked, discrete-threshold electromyography in the extreme lateral interbody fusion approach. J Neurosurg Spine 14:3137,2011

20. Uribe JS, Arredondo N, Dakwar E, Vale FL: Defining the safe working zones using the minimally invasive lateral retroperitoneal transpsoas approach: an anatomical study. $\mathbf{J}$ Neurosurg Spine 13:260-266, 2010

21. Uribe JS, Deukmedjian AR, Mummaneni PV, Fu KM, Mundis GM Jr, Okonkwo DO, et al: Complications in adult spinal deformity surgery: an analysis of minimally invasive, hybrid, and open surgical techniques. Neurosurg Focus 36(5):E15, 2014

22. Uribe JS, Kolla J, Omar H, Dakwar E, Abel N, Mangar D, et al: Brachial plexus injury following spinal surgery. J Neurosurg Spine 13:552-558, 2010

23. Uribe JS, Vale FL, Dakwar E: Electromyographic monitoring and its anatomical implications in minimally invasive spine surgery. Spine (Phila Pa 1976) 35 (26 Suppl):S368-S374, 2010

24. Voyadzis JM, Felbaum D, Rhee J: The rising psoas sign: an analysis of preoperative imaging characteristics of aborted minimally invasive lateral interbody fusions at L4-5. J Neurosurg Spine 20:531-537, 2014

25. Yusof MI, Nadarajan E, Abdullah MS: The morphometric study of 13-L4 and L4-L5 lumbar spine in Asian population using magnetic resonance imaging: feasibility analysis for transpsoas lumbar interbody fusion. Spine (Phila Pa 1976) 39:E811-E816, 2014

\section{Disclosures}

Drs. Narita, Takatori, Ogura, and Kubo and Nantan General Hospital have applied for a patent for the finger electrode.

\section{Author Contributions}

Conception and design: Arai, Narita, Takatori. Acquisition of data: Narita, Takatori, Hayashida. Analysis and interpretation of data: Narita. Drafting the article: Narita. Critically revising the article: Narita. Reviewed submitted version of manuscript: Narita. Statistical analysis: Narita, Takatori. Administrative/technical/ material support: Arai, Takatori, Nagae, Ogura. Study supervision: Arai, Takatori, Nagae, Tonomura, Ogura, Fujiwara, Kubo.

\section{Supplemental Information \\ Videos \\ Video 1. https://vimeo.com/155682703.}

\section{Correspondence}

Yuji Arai, Department of Orthopaedics, Graduate School of Medical Science, Kyoto Prefectural University of Medicine, Kawaramachi-Hirokoji, Kamigyo-ku, Kyoto 602-8566, Japan. email: yarai89046@nike.eonet.ne.jp. 